\title{
New Developments in Nonrelativistic Gravity
}

\author{
Eric A. Bergshoeff ${ }^{1}$, Jan Rosseel ${ }^{2}$ and Thomas Zojer ${ }^{3}$
}

${ }^{1}$ Centre for Theoretical Physics, University of Groningen, Nijenborgh 4, 9747 AG Groningen, The Netherlands

${ }^{2}$ Albert Einstein Center for Fundamental Physics, University of Bern, Sidlerstrasse 5, 3012 Bern, Switzerland

${ }^{3}$ Centro de Estudios Cientficos (CECs), Av. Arturo Prat 514, Valdivia, Chile

emails: E.A.Bergshoeff@rug.nl, rosseelj@gmail.com and th.zojer@gmail.com

\begin{abstract}
In this presentation we give a short overview of Newton-Cartan geometry and gravity and some recent results about its matter couplings.
\end{abstract}




\section{Introduction}

It is well known that in Newtonian gravity free-falling frames are connected by the Galilean symmetries which consist of (constant) time translations, spatial translations, spatial rotations and Galilean boosts. These Galilean boosts rotate space into time but not the other way around since Newtonian time is absolute. In such free-falling frames one does not experience any gravitational force. Such a force is only felt in frames that are not free-falling. For instance, in a (non-rotating) earth-based frame, that is in constant acceleration with respect to a free-falling frame, one experiences a gravitational force force that is described by the Newton potential satisfying a Poisson equation. Of course, in a different frame than an earth-based frame, one experiences a different gravitational force that, in principle, can be calculated by relating that frame to a given earth-based or free-falling frame. However, a truly frame-independent formulation of Newtonian gravity was never given by Newton and his followers. The reason for this is that in order to give such a frame-independent formulation one needs a piece of mathematics that was not yet developed around that time. It was only in the middle of the 19th century that Riemann developed the required tools geometry that is now called geometry.

When Einstein invented his General Relativity theory in 1915 he achieved two things. First of all, he made his way of describing gravity consistent with the Special Relativity theory he had developed 10 years earlier by making use of the geometry of spacetime to give a proper description of gravity. In this way he built in a delay effect that avoided the instantaneous gravitational force of Newton. But most importantly, and this took Einstein many years of hard work to achieve, he presented his equations in a frame-independent way. For this, he needed the Riemannian geometry mentioned above and communicated to him by his friend Albert Grossmann. Free-falling frames in Einstein's theory are connected by the Poincare symmetries. These differ from the Galilean symmetries only as far as the boosts are concerned. Unlike the Galilean boosts the Lorentzian boosts rotate space into time and time into space: the concept of time is relative in Einstein's theory. Furthermore, to obtain a frame-independent formulation Einstein introduced a symmetric tensor field to describe the gravitational force. This field replaces the Newton potential and describes geometrical distances in the Riemannian spacetime manifold.

It was only 8 year later that Elie Cartan did for Newtonian gravity what Einstein had achieved for relativistic gravity. The formulation of Newtonian gravity in an arbitrary frame goes under the name of Newton-Cartan (NC) gravity. This NC gravity theory contains more fields that just the Newton potential. The formulation given by Newton, with a Newton potential in an earth-based frame, can easily be obtained from the general formulation by an appropriate gauge-fixing of the gravitational fields such that one is left with the Newton potential as the only non-zero field. The geometry Cartan was using is called Newton-Cartan geometry. This NC geometry differs from the Riemannian geometry used by Einstein in the sense that it is a foliated 
geometry with an absolute time direction.

Given NC geometry and gravity the question arises: why should we study non-relativistic gravity? There are two main reasons why NC gravity has seen a return of interest in recent years. First of all, it arises in the context of the so-called holographic principle which states that all the information about a gravitational theory in a given volume can be encoded by a different so-called field theory that lives on the surface surrounding this volume. This holographic principle has found a precise mathematical framework in string theory where it goes under the name of the so-called AdS/CFT correspondence. This is a special situation where the gravity theory lives in a maximally symmetric spacetime with a negative cosmological constant, a socalled Anti-de Sitter (AdS) spacetime, and where the field theory is a special so-called conformal field theory (CFT). In recent years people have studied also non-AdS holography to understand the validity and the basic principles underlying the holographic principle. One of the simplest deviations of AdS is a Lifshitz spacetime which has less symmetries than AdS. Correspondingly, it has been found that at the field theory side the relativistic scale invariance of the CFT is broken to a non-relativistic scale invariance corresponding to a field theory that couples to an extension of $\mathrm{NC}$ geometry with so-called 'twistless torsion' [1]. Quite independently, NG geometry has found recently applications in the condensed matter physics community. Independent of any holographic interpretation one works here with an Effective Field Theory (EFT) coupled to NC geometry to describe general features of models such as the fractional quantum Hall effect [2], chiral superfluids and simple fluids. The coupling to NC gravity means that one uses an arbitrary frame formulation in which general features are visible. One could compare this with the Coriolis force that is not visible in a non-rotating earth-based frame but can only be observed in a more general (rotating) frame.

Having the above motivation in mind we will first show in the section 2 how NC gravity can be obtained via a kind of gauging procedure from the centrally extended Galileai algebra which is called the Bargmann algebra. Next, in section 3, we will discuss some recent results on matter couplings.

\section{Newton-Cartan from gauging Bargmann}

Let us first remind ourselves how to obtain Einstein gravity via a kind of gauging procedure from the Poincare algebra. In general relativity all freefalling frames are connected by the following Poincare symmetries:

- space-time translations: $\quad \delta x^{\mu}=\xi^{\mu}$,

- Lorentz transformations: $\delta x^{\mu}=\lambda^{\mu}{ }_{\nu} x^{\nu}$.

In arbitrary frames the gravitational force is described by the metric field. Instead of a metric, it is convenient to use an equivalent Vierbein formulation, with Vierbein field $e_{\mu}{ }^{A}(\mu=0,1,2,3 ; A=0,1,2,3)$ since these Vierbeine are naturally related to the gauge fields of Poincare translations. 
In the non-relativistic case all free-falling frames are connected by the Galilean symmetries:

- time translations: $\quad \delta t=\xi^{0}$,

- space translations: $\quad \delta x^{i}=\xi^{i}, \quad i=1,2,3$,

- spatial rotations: $\delta x^{i}=\lambda^{i}{ }_{j} x^{j}$,

- Galilean boosts: $\quad \delta x^{i}=\lambda^{i} t$.

They are identical to the Poincare symmetries except for the Galilean boosts which differ from the Lorentzian boosts as we discussed in the Introduction.

It is important to distinguish Newtonian gravity from Newton-Cartan gravity. Newtonian gravity is valid in frames of constant acceleration with respect to free-falling frames and is described by a single Newton potential $\Phi(x)$. On the other hand. Newton-Cartan gravity is valid in arbitrary frames but need more fields to describe the gravitational force. To be precise, the required fields are a so-called temporal Vierbein $\tau_{\mu}(x)$ and a spatial Vierbein $e_{\mu}{ }^{a}(x)$. Since these two fields together form a $4 \times 4$ matric $\left\{\tau_{\mu}, e_{\mu}{ }^{a}\right\}$ one would think that these fields suffice. Surprisingly, one needs one more field to describe Newton-Cartan gravity, namely a vector field $m_{\mu}(x)$.

One way to understand why this extra field is needed is to compare a freely moving relativistic particle with its non-relativistic counterpart. On the one hand a relativistic particle is described by the action

$$
S_{\text {relativistic }}=-m \int d \tau \sqrt{-\eta_{\mu \nu} \dot{x}^{\mu} \dot{x}^{\nu}} \quad \mu=0,1,2,3,
$$

where $x^{\mu}(\tau)$ are the embedding coordinates. Clearly, the Lagrangian corresponding to this action is invariant under the Poincare symmetries. On the other hand, a non-relativistic particle is dcescribed by the action

$$
S_{\text {non-relativistic }}=\frac{m}{2} \int \frac{\dot{x}^{i} \dot{x}^{j} \delta_{i j}}{\dot{t}} d \tau \quad i=1,2,3 .
$$

In this case the Lagrangian corresponding to this action is not invariant under Galilean boosts. Instead, the Lagrangian transforms with a total derivative as follows:

$$
\delta L_{\text {non-relativistic }}=\frac{d}{d \tau}\left(m x^{i} \lambda^{j} \delta_{i j}\right) .
$$

Although the action is invariant, the non-invariant Lagrangian leads to modified Noether charges which induce a central extension of the underlying Galilei algebra. One thus ends up with the Bargmann algebra where the gauge field of the extra central charge transformation is the vector field $m_{\mu}(x)$.

Before gauging the Bargmann algebra it is of interest to compare gaugings and Inönü-Wigner contractions of algebras and taking the non-relativistic limit of gravity. We have indicated the relations between these different manipulations below. 


$\begin{array}{llr}\text { Poincare } \otimes \mathrm{U}(1) & \stackrel{\text { General relativity } \otimes \mathrm{U}(1)}{\longrightarrow} & \Downarrow \text { non-relativistic limit } \\ \text { contraction } \Downarrow & & \text { 'gauging' } \\ \text { Bargmann } & \stackrel{\text { 'gauging' }}{\longrightarrow} & \text { Newton-Cartan gravity }\end{array}$

We see that, in order to obtain the Bargmann algebra from a contraction of the Poincare algebra we need first to extend the Poincare algebra with an additional U(1) generator, in order to account for the central charge generator which is present in the Bargmann algebra on top of the usual Galilei generators. This suggests that the non-relativistic limit of general relativity can only be taken in the presence of an additional vector field that corresponds to the extra $\mathrm{U}(1)$ generator. This non-relativistic limit should mimick the Inönü-Wigner contraction of the algebra.

We now return to the gauging of the Bargman algebra [3] which is based on a similar gauging procedure developed in the supergravity community many years ago [4]. Our starting point is the set of commutation relations defining the Bargmann algebra

$$
\begin{aligned}
{\left[J_{a b}, P_{c}\right] } & =-2 \delta_{c[a} P_{b]}, & {\left[J_{a b}, G_{c}\right] } & =-2 \delta_{c[a} G_{b]}, \\
{\left[G_{a}, H\right] } & =-P_{a}, & {\left[G_{a}, P_{b}\right] } & =-\delta_{a b} Z, \quad a=1,2, \ldots, d,
\end{aligned}
$$

where $\left\{H, P_{a}, J_{a b}, G_{a}, Z\right\}$ are the generators of time translations, space translations, spatial rotations, Galilean boosts and central charge trransformations, respectively. In this gauging procedure we associate to every generator/symmetry a gauge field, gauge parameters that are arbitrary functions of spacetime and covariant curvatures as indicated in the table below.

\begin{tabular}{|c|c|c|c|c|}
\hline symmetry & generators & gauge field & parameters & curvatures \\
\hline time translations & $H$ & $\tau_{\mu}$ & $\zeta\left(x^{\nu}\right)$ & $\mathcal{R}_{\mu \nu}(H)$ \\
space translations & $P^{a}$ & $e_{\mu}{ }^{a}$ & $\zeta^{a}\left(x^{\nu}\right)$ & $\mathcal{R}_{\mu \nu}{ }^{a}(P)$ \\
Galilean boosts & $G^{a}$ & $\omega_{\mu}{ }^{a}$ & $\lambda^{a}\left(x^{\nu}\right)$ & $\mathcal{R}_{\mu \nu}{ }^{a}(G)$ \\
spatial rotations & $J^{a b}$ & $\omega_{\mu}{ }^{a b}$ & $\lambda^{a b}\left(x^{\nu}\right)$ & $\mathcal{R}_{\mu \nu}{ }^{a b}(J)$ \\
central charge transf. & $Z$ & $m_{\mu}$ & $\sigma\left(x^{\nu}\right)$ & $\mathcal{R}_{\mu \nu}(Z)$ \\
\hline
\end{tabular}

Table 1: This table indicates for every symmetry the corresponding generators, gauge fields, local gauge parameters and covariant curvatures.

From the Table we see that besides a timelike Vierbein $\tau_{\mu}$ and a spatial Vierbein $e_{\mu}{ }^{a}$ there are two independent spin-connections fields $\left\{\omega_{\mu}^{a b}, \omega_{\mu}^{a}\right\}$ of spatial rotations and Galilean boosts, respectively, and a gauge field $m_{\mu}$ for the central charge transformations. Following general relativity, in order to make the spin-connection fields dependent we need to impose constraints on the curvatures. Unlike general relativity, the curvature $\mathcal{R}_{\mu \nu}(H)$ of time translations cannot play any role here since that curvature does not contain 
any of the two spin-connections fields. At this point the curvature $\mathcal{R}_{\mu \nu}(Z)$ of the central charge transformations comes to help since that curvature does contain the spin-connection field of the Galilean boosts. Independent of this we do set the curvature of time translations to zero since this defines the foliation of spacetime. We thus arrive at the following set of curvature constraints:

$$
\begin{array}{cc}
\mathcal{R}_{\mu \nu}{ }^{a}(P)=0, \quad \mathcal{R}_{\mu \nu}(Z)=0: & \text { solve for spin-connection fields } \\
\mathcal{R}_{\mu \nu}(H)=\partial_{[\mu} \tau_{\nu]}=0 \rightarrow \tau_{\mu}=\partial_{\mu} \tau: & \text { absolute time ('zero torsion') } \\
\mathcal{R}_{\mu \nu}{ }^{a b}(J) \neq 0: & \text { un-constrained off-shell } \\
\mathcal{R}_{0(a, b)}(G) \neq 0: & \text { un-constrained off-shell }
\end{array}
$$

Note that the zero torsion constraint (6) allows us to solve for the timelike Vierbein in terms of an arbitrary function $\tau\left(x^{\nu}\right)$ of the spacetime coordinates. Choosing $\tau\left(x^{\mu}\right)=t$ defines the time-coordinate $t$ to be the absolute time but there are other choices possible as well.

Following the standard gauging procedure one ends up with three independent gauge-fields $\left\{\tau_{\mu}, e_{\mu}{ }^{a}, m_{\mu}\right\}$ that transform under general coordinate transformations, with parameters $\xi^{\mu}$, as covariant vectors and under the other Bargmann symmetries as follows:

$$
\begin{aligned}
\delta \tau_{\mu} & =\xi^{\lambda} \partial_{\lambda} \tau_{\mu}+\partial_{\mu} \xi^{\lambda} \tau_{\lambda}, \\
\delta e_{\mu}{ }^{a} & =\xi^{\lambda} \partial_{\lambda} e_{\mu}{ }^{a}+\partial_{\mu} \xi^{\lambda} e_{\lambda}{ }^{a}+\lambda^{a}{ }_{b} e_{\mu}{ }^{b}+\lambda^{a} \tau_{\mu}, \\
\delta m_{\mu} & =\xi^{\lambda} \partial_{\lambda} m_{\mu}+\partial_{\mu} \xi^{\lambda} m_{\lambda}+\partial_{\mu} \sigma+\lambda_{a} e_{\mu}{ }^{a} .
\end{aligned}
$$

Furthermore, one may define two Galilean-invariant metrics

$$
\tau_{\mu \nu}=\tau_{\mu} \tau_{\nu}, \quad h^{\mu \nu}=e^{\mu}{ }_{a} e^{\nu}{ }_{b} \delta^{a b},
$$

one in the time direction and a separate one in the spatial directions. Note that the timelike metric is only defined with lower indices whereas the spatial metric is only defined with upper indices. Without the central charge vector field it is not possible to define a timelike metric with upper indices and a spatial metric with lower indices that is invariant under Galilean boosts. Such unwanted variations can only be canceled by adding $m_{\mu}$-dependent terms to these metrics.

Now that we have defined the symmetries of NC gravity in arbitrary frames it is easy to switch between frames. For instance, to go from the general frame formulation back to the free-falling frames only, one must impose the following gauge-fixing conditions eliminating all gravitational fields:

$$
\tau_{\mu}=\delta_{\mu}^{t}, \quad e_{t}^{a}=0, \quad e_{i}^{a}=\delta_{i}^{a}, \quad m_{\mu}=0 .
$$

This leads to the following non-relativistic Killing equations:

$$
\xi^{t}\left(x^{\mu}\right)=\zeta, \quad \xi^{i}\left(x^{\mu}\right)=\xi^{i}-\lambda^{i} t-\lambda^{i}{ }_{j} x^{j}, \quad \sigma\left(x^{\mu}\right)=\sigma-\lambda^{i} x^{i},
$$


whose most general solution is given by the Galilean symmetries connecting free-falling frames:

$$
\xi^{t}\left(x^{\mu}\right)=\zeta, \quad \xi^{i}\left(x^{\mu}\right)=\xi^{i}-\lambda^{i} t-\lambda^{i}{ }_{j} x^{j}, \quad \sigma\left(x^{\mu}\right)=\sigma-\lambda^{i} x^{i} .
$$

Instead, one could also go from general frames to frames with constant acceleration. In that case one has to impose less stringent gauge-fixing conditions in which the Newton potential survives as one of the components of the gravitational fields. This gauge-fixing automatically gives the correct transformation rule of the Newton potential under the Bargmann symmetries.

Sofar, we have only defined the kinematics of NC gravity. To define the dynamics we need to impose equations of motion. For this purpose we introduce the following equations:

$$
\begin{array}{rlcc}
\tau^{\mu} e^{\nu}{ }_{a} \mathcal{R}_{\mu \nu}{ }^{a}(G) & =0 & \mathbf{1} \\
e^{\nu}{ }_{a} \mathcal{R}_{\mu \nu}{ }^{a b}(J) & =0 & & \mathbf{a}+(\mathbf{a b}),
\end{array}
$$

where we have indicated at the right the representations of spatial rotations to which these equations belong. The first singlet equation reduces to the Poisson equation for the Newton potential after gauge-fixing to frames with constant acceleration. Note that, without the second equation, the first equation would not be invariant under Galilean boosts. The number of equations is the same as in general relativity but the number of the independent fields is not the same. Therefore, there is no obvious way to integrate the above $\mathrm{NC}$ equations of motion to an action.

\section{Adding Matter}

One way to add matter to $\mathrm{NC}$ gravity is to start from the relativistic answer and take the non-relativistic limit. In this way one obtains matter couplings from arbitrary contracting backgrounds [5]. ${ }^{1}$ As a bonus this also gives an elegant way to derive non-relativistic field theories from relativistic ones. In the figure below we have indicated how this works for Klein-Gordon versus Schrödinger.

$$
\text { scalar }+\mathrm{GR} \quad \stackrel{\text { 'limit' }}{\Longrightarrow} \text { Schrödinger }+\mathrm{NC}
$$

general frames $\Uparrow$

$$
\text { Klein-Gordon } \quad \stackrel{?}{\Longrightarrow} \quad \text { Schrödinger }
$$

Fig 1. This figure indicates how to obtain Schrödinger coupled to NC gravity from KleinGordon coupled to general relativity by taking a non-relativistic limit. It also indicates how, as a bonus, we can obtain pure Schrödinger from pure Klein-Gordon by switching between general and free-falling frames .

\footnotetext{
${ }^{1}$ For another recent and related discussion, see [6].
} 
We first define the non-relativistic limit of general relativity without matter by mimicking the Inönü-Wigner contraction of the corresponding algebra as much as possible. This contraction works as follows. Our starting point is the Poincare algebra plus an additional $\mathrm{U}(1)$ generator $\mathcal{Z}$ that commutes with all the Poincare generators:

$$
\left[P_{A}, M_{B C}\right]=2 \eta_{A[B} P_{C]}, \quad\left[M_{A B}, M_{C D}\right]=4 \eta_{[A[C} M_{D] B]} \text { plus } \mathcal{Z}
$$

Here $\left\{P_{A}, M_{A B}\right\}$ are the generators of spacetime translations and Lorentz generators, respectively. Next, we decompose $A=(0, a)$ and relate the Poincare $\otimes \mathrm{U}(1)$ generators $\left\{P_{0}, P_{a}, M_{a o}, M_{a b}\right\}$ to the Bargmann generators $\left\{H, P_{a}, G_{a}, J_{a b}, Z\right\}$ as follows

$$
\begin{aligned}
& P_{0}=\frac{1}{2 \omega} H+\omega Z, \quad \mathcal{Z}=\frac{1}{2 \omega} H-\omega Z, \quad A=(0, a), \\
& P_{a}=P_{a}, \quad M_{a b}=J_{a b}, \quad M_{a 0}=\omega G_{a},
\end{aligned}
$$

where we have introduced a contraction parameter $\omega$. In a second step, taking the limit $\omega \rightarrow \infty$, we obtain the Bargmann algebra including the following commutator containing the central charge generator $Z$ :

$$
\left[P_{a}, G_{b}\right]=\delta_{a b} Z
$$

Inspired by the above Inönü-Wigner contraction we now define the nonrelativistic limit of general relativity as follows. We first introduce, on top of the Vierbein field, a vector field $M_{\mu}$ with $\partial_{[\mu} M_{\nu]}=0$. Next, we relate the relativistic gauge fields $\left\{E_{\mu}{ }^{A}, M_{\mu}\right\}$ to the non-relativistic gauge fields $\left\{\tau_{\mu}, e_{\mu}{ }^{a}, m_{\mu}\right\}$ as follows:

$$
E_{\mu}{ }^{0}=\omega \tau_{\mu}+\frac{1}{2 \omega} m_{\mu}, \quad M_{\mu}=\omega \tau_{\mu}-\frac{1}{2 \omega} m_{\mu}, \quad E_{\mu}{ }^{a}=e_{\mu}{ }^{a} .
$$

This implies for the inverse Vierbein fields the following relation:

$$
E^{\mu}{ }_{a}=e^{\mu}{ }_{a}-\frac{1}{2 \omega^{2}} \tau^{\mu} e^{\rho}{ }_{a} m_{\rho}+\mathcal{O}\left(\omega^{-4}\right) \text { and similar for } E^{\mu}{ }_{0} .
$$

The definitions of the non-relativistic inverse fields $\left\{\tau^{\mu}, e^{\mu}{ }_{a}\right\}$ we have used here can be found in [3].

In a second step we now take the limit $\omega \rightarrow \infty$. In this way we obtain the correct non-relativistic transformation rules (9) and the equations of motion (13). Note that the standard textbooks on general relativity usually go straight from general relativity to Newtonian gravity skipping the general frame formulation of $\mathrm{NC}$ gravity.

As an example we consider a complex scalar of mass $M$ with Lagrangian given by

$$
\begin{gathered}
E^{-1} \mathcal{L}_{\text {rel }}=-\frac{1}{2} g^{\mu \nu} D_{\mu} \Phi^{*} D_{\nu} \Phi-\frac{M^{2}}{2} \Phi^{*} \Phi \quad \text { with } \\
D_{\mu} \Phi=\partial_{\mu} \Phi-\mathrm{i} M M_{\mu} \Phi, \quad \delta \Phi=\mathrm{i} M \Lambda \Phi .
\end{gathered}
$$


In a free-falling frame this Lagrangian reduces to the standard Klein-Gordon Lagrangian.. Note that $M_{\mu}$ is not electromagnetic gauge field. The mass $M$ is not equal to the electric charge $q$. Instead the gauge field $M_{\mu}$ couples to the current expressing the conservation of \# particles - \# anti-particles.

We now take the non-relativistic limit of general relativity as defined above together with $M=\omega m, \Phi \rightarrow \sqrt{\frac{\omega}{m}} \phi$. This leads us to the following Schrödinger Lagrangian coupled to NC gravity:

$$
\begin{gathered}
e^{-1} \mathcal{L}_{\text {Schroedinger }}=\left[\frac{\mathrm{i}}{2}\left(\Phi^{*} \tilde{D}_{0} \Phi-\Phi \tilde{D}_{0} \Phi^{*}\right)-\frac{1}{2 m}\left|\tilde{D}_{a} \Phi\right|^{2}\right] \text { with } \\
\tilde{D}_{\mu} \Phi=\partial_{\mu} \Phi+\mathrm{i} m m_{\mu} \Phi, \quad \delta \Phi=\xi^{\mu} \partial_{\mu} \Phi-\mathrm{i} m \sigma \Phi .
\end{gathered}
$$

In a free-falling frame this is the standard Shrödinger Lagrangian. Note that the non-relativistic gauge field $m_{\mu}$ couples to the current that expresses the conservation of \# particles only. Intuitively, the extra vector gauge field takes care of the infinities that occur if you switch between a Lagrangian with 2 time derivatives and a Lagrangian with one time derivative.

\section{Acknowledgements}

One of us, E.B., wishes to thank the organizers of the SQS'2015 workshop for providing a stimulating atmosphere and offering a diverse scientific programme.

\section{References}

[1] Christensen M.H., Hartong J., Obers N.A. and Rollier B Torsional Newton-Cartan Geometry and Lifshitz Holography Phys. Rev. D 89 (2014) 061901 doi:10.1103/PhysRevD.89.061901 [arXiv:1311.4794 [hepth]].

[2] Son D.T. Newton-Cartan Geometry and the Quantum Hall Effect arXiv:1306.0638 [cond-mat.mes-hall].

[3] Andringa R., Bergshoeff E., Panda S. and de Roo M. Newtonian Gravity and the Bargmann Algebra Class. Quant. Grav. 28 (2011) 105011 doi:10.1088/0264-9381/28/10/105011 [arXiv:1011.1145 [hep-th]].

[4] Chamseddine A.H. and West P.C. Supergravity as a Gauge Theory of Supersymmetry, Nucl. Phys. B 129 (1977) 39. doi:10.1016/05503213(77)90018-9

[5] Bergshoeff E., Rosseel J. and Zojer T. Non-relativistic fields from arbitrary contracting backgrounds, arXiv:1512.06064 [hep-th].

[6] Jensen K., and Karch A. Revisiting non-relativistic limits, JHEP 1504 (2015) 155 doi:10.1007/JHEP04(2015)155 [arXiv:1412.2738 [hep-th]]. 